\title{
Analyse d'ensemble des complications de la COVID-19 : étude d'auto-appariement pré- et post-exposition
}

\author{
William Murk PhD MSP, Monica Gierada MSP, Michael Fralick MD PhD MSc, Andrew Weckstein BA, Reyna Klesh MSc, \\ Jeremy A. Rassen DSC
}

- Citation : CMAJ 2021 January 4;193:E10-8. doi : 10.1503/cmaj.201686-f; diffusion hâtive le 8 décembre 2020

Voir la version anglaise de l'article ici : www.cmaj.ca/lookup/doi/10.1503/cmaj.201686

\section{RÉSUMÉ}

CONTEXTE : De nombreuses études sur les complications de la maladie à coronavirus 2019 (COVID-19) ont reposé sur des séries de cas et de petites cohortes qui ne permettaient pas d'établir un lien causal avec la COVID-19 ni d'estimer les risques dans les différents milieux de soins. Nous avons voulu étudier toutes les complications possibles de la COVID-19 afin de confirmer les complications précédemment déclarées et d'identifier de potentielles complications encore inconnues.

MÉTHODES : À partir des données sur les demandes de remboursement de frais médicaux aux États-Unis, nous avons comparé la fréquence de tous les codes de diagnostic de la Classification internationale des maladies, $10^{e}$ révision, modification clinique (CIM-10-MC) enregistrés avant et après le déclenchement de la pandémie de COVID-19 dans un modèle d'auto-appariement pré- et post-exposition. Nous avons inclus les patients ayant reçu un diagnostic de COVID-19 entre le $1^{\text {er }}$ mars 2020 et le 30 avril 2020, et calculé les estimations de risque et les rapports de cotes (RC) pour le lien avec la COVID-19 de chaque code de diagnostic de la CIM-10-MC.

RÉSULTATS : Sur les 1724 codes de diagnostic de la CIM-10-MC attribués à 70288 patients atteints de COVID-19, 69 étaient significativement liés à la COVID-19. Les diagnostics étroitement liés à la COVID-19 et comportant un risque absolu élevé comprenaient la pneumonie virale (RC 177,63; intervalle de confiance [IC] à $95 \%$ 147,19-214,37; risque absolu $27,6 \%$ ), l'insuffisance respiratoire (RC 11,36; IC à $95 \% 10,74-$
12,02 ; risque absolu 22,6\%), l'insuffisance rénale aiguë (RC 3,50; IC à $95 \%$ $3,34-3,68$; risque absolu $11,8 \%$ ) et la sepsie (RC 4,23; IC à $95 \% 4,01-4,46$; risque absolu $10,4 \%)$. Les diagnostics étroitement liés à la COVID-19, mais comportant un risque absolu faible comprenaient la myocardite (RC 8,17 ; IC à $95 \% 3,58-18,62$; risque absolu $0,1 \%)$, la coagulation intravasculaire disséminée (RC 11,83; IC à $95 \%$ 5,2626,62 ; risque absolu $0,1 \%$ ) et le pneumothorax (RC 3,38; IC à 95\% 2,68-4,26; risque absolu $0,4 \%$ ).

INTERPRÉTATION : Nous avons confirmé et établi les estimations du risque de plusieurs complications de la COVID-19. Ces résultats pourraient orienter le pronostic, les décisions thérapeutiques et les conseils aux patients.

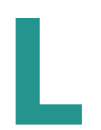

e coronavirus du syndrome respiratoire aigu sévère 2 (SRAS-CoV-2) est une nouvelle souche de coronavirus qui est à l'origine de la pandémie de maladie à coronavirus 2019 (COVID-19). En date du 20 novembre 2020, plus de 50 millions de personnes avaient reçu un diagnostic de COVID-19 dans le monde ${ }^{1}$. Le spectre clinique de la maladie est vaste et peut aller des symptômes de rhume à l'insuffisance respiratoire, voire au décès ${ }^{2}$. La majeure partie des patients ont des symptômes bénins et n'ont pas besoin d'être hospitalisés, mais jusqu'à $20 \%$ présentent une forme grave de la maladie et nécessitent une hospitalisation, habituellement en raison d'une hypoxie secondaire à une pneumonie ${ }^{3}$.
Des études montrent aussi que la COVID-19 est associée à diverses complications non respiratoires, notamment endothéliales, thrombotiques, cardiaques, inflammatoires, neurologiques et autres ${ }^{4-9}$. Le lien de cause à effet n'a pas été clairement établi pour ces complications, puisque bon nombre de ces signalements proviennent de rapports de cas qui sont sujets à des biais de publication incompatibles avec une estimation des risques, ou d'études de cohorte qui, souvent, ne fournissent pas ce type d'estimation.

Une autre stratégie de détection des complications potentielles de la COVID-19 consiste à étudier toutes les complications possibles à l'aide des codes de diagnostic de la Classification internationale des maladies, $10^{e}$ révision, modification clinique 
(CIM-10-MC) afin de mettre au jour celles qui n'ont pas été signalées et de confirmer celles qui l'ont déjà été. L'objectif de notre étude était d'analyser tous les diagnostics associés à la COVID-19, de déterminer ceux qui pourraient représenter une complication et de présenter le risque absolu et le rapport de cotes des complications relevées.

\section{Méthodes}

\section{Source des données}

Cette étude repose sur les réclamations pour frais médicaux anonymisées de l'ensemble de données de la plateforme américaine HealthVerity Marketplace. Ces données représentent les réclamations adressées à des régimes d'assurance santé nationaux qui regroupent les principaux types de payeurs (régimes privés, Medicaid et Medicare). Elles incluent les réclamations non réglées, générées presque en temps réel par les systèmes de gestion des cabinets, les systèmes de facturation et les centres de réclamations, ainsi que les réclamations réglées, générées par les assureurs et les payeurs. Les données concernant les réclamations non réglées peuvent être saisies dans les jours qui suivent une consultation, tandis que les données des réclamations réglées demandent plus de temps. Les réclamations non réglées offrent donc des données plus récentes, tandis que les réclamations réglées donnent un aperçu plus complet des interactions d'un patient avec le système de santé. Ces sources de données sont décrites plus en détail à l'annexe 1, Méthodes, accessible en anglais au www.cmaj. ca/lookup/doi/10.1503/cmaj.201686/tab-related-content. Les réclamations étaient accessibles pour les dates de service allant du $1^{\text {er }}$ novembre 2019 au 30 mai 2020.

\section{Population étudiée}

Nous avons inclus tous les patients qui ont eu au moins une consultation médicale en lien avec la COVID-19, définie par les codes de diagnostic U07.1 (COVID-19, virus identifié) ou B97.29 (autre coronavirus ayant causé des maladies classées ailleurs) de la CIM-10-MC, entre le $1^{\text {er }}$ mars 2020 et le 30 avril 2020. Le 30 avril était la dernière date d'admissibilité permettant un suivi d'un mois auprès de tous les patients. Les codes ont été sélectionnés conformément au guide des Centers for Disease Control and Prevention (CDC) des États-Unis à cet effet pour les cas confirmés de COVID-1910,11. La date de la première réclamation admissible durant la période à l'étude est la date initiale. Les patients qui s'étaient vu attribuer les codes U07.1, B97.29 ou B97.21 (coronavirus associé au SRAS) avant la date initiale et ceux dont on n'avait pas la date de naissance ont été exclus. Les patients ayant reçu le code Z03.818 dans la période allant de 7 jours avant à 30 jours après la date initiale ont aussi été exclus parce que ce code indiquait qu'une exposition au SRAS-CoV-2 a été écartée ${ }^{10}$. Pour être certains que tous les patients analysés étaient observables avant leur diagnostic de COVID-19, nous avons exclu ceux pour lesquels aucune réclamation n'avait été faite dans la période allant de 120 à 30 jours avant la date initiale. Enfin, nous avons analysé des sous-groupes de patients traités seulement en clinique externe, de patients hospitalisés et de patients hospitalisés aux soins intensifs (annexe 1, Méthodes).

\section{Modèle d'étude}

Nous avons utilisé un modèle d'auto-appariement pré- et postexposition ${ }^{12}$ pour recenser les diagnostics dont le risque augmentait après l'infection par le virus de la COVID-19. Ce modèle atténue les possibilités de confusion non mesurées en comparant l'état de santé d'un patient au moment du diagnostic de COVID-19 ("période de risque ») à son état de santé antérieur ("période de référence »). Tout accroissement du risque d'un problème de santé était jugé comme étant probablement attribuable à la COVID-19 ou à son traitement.

Pour saisir l'activité liée à la COVID-19 juste avant l'établissement du diagnostic (annexe 1, figure 2), nous avons défini la période de risque comme suit : de 7 jours avant à 30 jours après la date initiale. Nous avons aussi défini la période de référence comme suit : du $120^{\mathrm{e}}$ au $30^{\mathrm{e}}$ jour avant la date initiale. Nous avons choisi cette fenêtre de 90 jours parce qu'elle était suffisante pour rendre compte de la plupart des maladies chroniques tout en permettant de rendre également compte de l'état de santé de base de tous les patients. Nous avons considéré les 23 jours entre la période de référence et la période de risque comme étant une période d'état inconnu à l'égard de la COVID-19 (annexe 1, figure 2) et exclu cette valeur personne-temps de l'analyse. Nous avons aussi procédé à des analyses de sensibilité pour mesurer l'effet de la durée de la période de référence en examinant les cas où les périodes de référence et de risque étaient de même durée (du $68^{\mathrm{e}}$ au $30^{\mathrm{e}}$ jour avant la date initiale; 38 jours en tout). Le modèle de l'étude est illustré à l'annexe 1, figure 3.

\section{Analyse statistique}

Pour repérer les codes de la CIM-10-MC associés à la COVID-19, nous avons apparié les observations concernant chaque patient pour la période de référence et pour la période de risque, nous avons calculé les rapports de cotes (RC) pour estimer la solidité du lien entre le code et la COVID-19 et nous avons obtenu les valeurs $p$ au moyen du test McNemar ${ }^{13}$. Nous avons utilisé des tests exacts si le nombre de paires discordantes était inférieur à 25 . Les codes de la CIM-10-MC sont classés par systèmes anatomiques et par maladie; ces catégories sont désignées par 3 caractères qui peuvent être suivis d'un maximum de 4 chiffres pour plus de précision ${ }^{14,15}$. Pour notre analyse principale, nous nous sommes penchés sur les 1724 codes de diagnostic de la CIM-10-MC présents dans l'ensemble de données, regroupés par catégories (p. ex., «R43 - Troubles de l'odorat et du goût »). Pour toute catégorie de codes jugée comme ayant un lien significatif avec la COVID-19 à l'analyse principale, nous avons procédé à une analyse des codes individuels (p. ex., «R43.0 - Anosmie »). Pour notre analyse secondaire, nous avons analysé l'ensemble des 64931 codes individuels de la CIM-10-MC. Nous avons appliqué des seuils de significativité après correction de Bonferroni, définis comme une erreur de type 1 nominale de 0,05 divisée par le nombre de codes analysés (2.9E-05 et 7.7E-07 pour les analyses principale et secondaires, respectivement $)^{16}$. Nous avons exclu les codes utilisés pour identifier les cas (B97 et U07). Une complication potentielle de la COVID-19 correspondait à tout code dont le risque augmentait avec la COVID-19 selon le seuil de significativité après correction de Bonferroni. 
Nous avons aussi calculé le risque absolu de recevoir un nouveau diagnostic pour chaque code de la CIM-10-MC une fois le diagnostic de COVID-19 posé. Il s'agissait du pourcentage de patients s'étant vu attribuer un code durant la période de risque par rapport aux patients qui n'avaient pas reçu ce code durant la période de référence (annexe 1, figure 3).

\section{Approbation éthique}

Cette étude a été approuvée en vertu de dispositions relatives à l'exemption par le New England Institutional Review Board (no 1-9757-1).

\section{Résultats}

Nous avons recensé 70288 patients ayant reçu un diagnostic de COVID-19 (tableau 1). Les patients exclus sont décrits à l'annexe 1, figure 4. Parmi ces patients, 53,4\% ont été hospitalisés et $4,7 \%$, admis aux soins intensifs. L'âge médian était de 65 ans, et $55,8 \%$ des personnes étaient de sexe féminin. Les 5 principaux États de résidence des patients étaient New York $(19,2 \%)$, le New Jersey (10,2\%), le Michigan (9,3\%), la Pennsylvanie $(7,5 \%)$ et l'Illinois (7,2\%). Les nombres médians des codes de diagnostic pour les périodes de référence et de risque sont présentés à l'annexe 1 , tableau 1 . Les estimations de la prévalence des maladies susceptibles d'influer sur le risque de COVID19 ou sur ses séquelles sont présentées au tableau 1.

\section{Identification des complications potentielles de la COVID-19}

Parmi les 1724 codes de diagnostic étudiés par catégories dans notre analyse principale, nous avons recensé 69 codes dont le risque augmentait avec la COVID-19 à un seuil de significativité statistique après correction de Bonferroni. Les systèmes respiratoires et circulatoires étaient les systèmes physiologiques le plus largement affectés (figure 1). Les rapports de cotes des complications potentielles de la COVID-19 sont présentés à la figure 2 .

Pour l'appareil respiratoire, les complications dont le code était le plus étroitement lié à la COVID-19 comprenaient la pneumonie (p. ex., pneumonie virale RC 177,63; IC à 95\% 147,19214,37), le syndrome de détresse respiratoire aiguë (SDRA) (RC 41,60; IC à $95 \%$ 33,00-52,45), l'infection aiguë des voies respiratoires inférieures (RC 28,77; IC à $95 \%$ 23,75-34,84), l'insuffisance respiratoire (RC 11,36; IC à $95 \% 10,74-12,02$ ) et le pneumotho$\operatorname{rax}(\mathrm{RC} 3,38$; IC à $95 \%$ 2,68-4,26). Pour l'appareil circulatoire, l'arrêt cardiaque (RC 10,43; IC à $95 \%$ 8,23-13,21) et la myocardite aiguë (RC 8,17; IC à $95 \%$ 3,58-18,62) présentaient le lien le plus étroit. Parmi les troubles hématologiques, la coagulation intravasculaire disséminée (RC 11,83; IC à $95 \%$ 5,26-26,62) était le plus étroitement associée, et parmi les troubles rénaux, l'insuffisance rénale aiguë (RC 3,50; IC à $95 \%$ 3,34-3,66) a été associée à la COVID-19. Les troubles de l'odorat et du goût (RC 14,62; IC à $95 \%$ 10,06-21,25) y ont également été étroitement associés. Des détails sur toutes les complications potentielles de la COVID-19 détectées dans l'analyse principale, de même que sur les codes dont la fréquence a augmenté avec une significativité statistique nominale sont fournis à l'annexe 1, tableau 2.
Les codes dont la fréquence a significativement diminué avec la COVID-19 sont présentés à l'annexe 1 , tableau 3. Des détails sur les 69 complications potentielles de la COVID-19 examinées à l'échelle des codes individuels sont fournis à l'annexe 1 , tableau 4.

L'analyse secondaire de l'ensemble des 64931 diagnostics de la CIM-10-MC (codes individuels) a mis en évidence 23 codes additionnels dont la fréquence a significativement augmenté avec la COVID-19 (annexe 1, tableau 5), y compris les codes de l'entérite virale (RC 18,00, IC à 95\% 8,96-36,16), de l'acidocétose (due au diabète de type $2-\mathrm{RC} 3,12$; IC à $95 \% 2,50-3,89$ ) et la rhabdomyolyse (RC 1,96; IC à $95 \%$ 1,65-2,33).

\section{Risque de complications potentielles de la COVID-19}

Parmi les complications repérées ayant un code associé à l'appareil respiratoire, celles dont le risque était le plus élevé comprenaient la pneumonie (p. ex., pneumonie virale $27,6 \%$ et $81,0 \%$ chez tous les patients et les patients aux soins intensifs, respectivement), l'insuffisance respiratoire $(22,6 \%$ et $75,3 \%$, respectivement) et le SDRA (4,3\% et $26,0 \%$, respectivement). Parmi les complications ayant un code associé à l'appareil circulatoire, celles dont le risque était le plus élevé étaient la fibrillation ou le flutter auriculaires $(4,6 \%$ et $16,1 \%$ chez tous les patients et les patients aux soins intensifs, respectivement), l'hypotension (3,8\% et $17,3 \%$, respectivement) et l'infarctus aigu du myocarde ( $1,8 \%$ et $8,1 \%$, respectivement). Le risque d'insuffisance rénale aiguë était de $11,8 \%$ chez tous les patients et de $50,7 \%$ chez les patients aux soins intensifs, et le risque d'autre sepsie était de $10,4 \%$ et $54,1 \%$, respectivement. Le risque d'autres affections du cerveau (encéphalopathie et autre; annexe 1, tableau 4) était de $4,9 \%$ et de $24,9 \%$, respectivement.

La figure 3 compare les estimations du risque aux estimations des RC et montre que les problèmes de santé les plus graves et le plus étroitement associés à la COVID-19 sont la pneumonie virale, l'insuffisance respiratoire, la sepsie ou la réponse inflammatoire systémique, l'insuffisance rénale aiguë et le SDRA. Les risques de présenter ces problèmes de santé selon l'âge et l'état de santé au moment de l'admission sont présentés à la figure 4 et à l'annexe 1 , tableau 6 . Les estimations du risque global pour la majorité des autres complications étaient relativement faibles, même pour les complications étroitement associées à la COVID19 , comme le pneumothorax $(0,4 \%$ et $2,6 \%$ de risque absolu chez tous les patients et les patients aux soins intensifs, respectivement), la coagulation intravasculaire disséminée $(0,1 \%$ et $0,9 \%$, respectivement) et la myocardite aiguë $(0,1 \%$ et $0,5 \%$, respectivement). L'annexe 1 présente le risque de toutes les complications potentielles de la COVID-19 repérées à l'analyse principale, calculé chez tous les patients (tableau 2), et chez les patients classés selon l'âge (tableau 7), selon le sexe (tableau 8) et selon le statut d'hospitalisation (tableau 9).

\section{Analyse de sensibilité}

Notre analyse de sensibilité pour évaluer l'effet d'une plus courte période de référence, soit 30 jours, a révélé une fréquence apparemment plus élevée de plusieurs maladies 
Tableau 1 : Caractéristiques démographiques et maladies préexistantes des patients atteints de la COVID-19*

\begin{tabular}{|c|c|c|c|c|}
\hline Caractéristiques & $\begin{array}{c}N^{\text {bre }}(\%) \dagger \text { parmi } \\
\text { tous les patients } \\
\quad n=70288\end{array}$ & $\begin{array}{c}\mathbf{N}^{\text {bre }}(\%) \dagger \text { parmi les } \\
\text { patients } \\
\text { ambulatoires } \neq \\
n=32745\end{array}$ & $\begin{array}{c}\mathrm{N}^{\mathrm{bre}}(\%) \dagger \text { parmi les } \\
\text { patients } \\
\text { hospitalisés§ } \\
n=37543\end{array}$ & $\begin{array}{c}\mathrm{N}^{\text {bre }}(\%) \dagger \text { parmi les } \\
\text { patients aux soins } \\
\text { intensifs } \uparrow \\
n=3269\end{array}$ \\
\hline Pourcentage du total, $n=70288$ & 100 & 46,6 & 53,4 & 4,7 \\
\hline Âge médian, ans (EI) & $65(51-77)$ & $56(41-70)$ & $71(59-81)$ & $69(59-78)$ \\
\hline \multicolumn{5}{|l|}{ Groupe d'âge, ans } \\
\hline 0 à $<20$ & $1722(2,4)$ & $1387(4,2)$ & $335(0,9)$ & $81(2,5)$ \\
\hline $20 \mathrm{à}<30$ & $3212(4,6)$ & $2582(7,9)$ & $630(1,7)$ & $53(1,6)$ \\
\hline 30 à < 40 & $4859(6,9)$ & $3605(11,0)$ & $1254(3,3)$ & $99(3,0)$ \\
\hline 40 à < 50 & $6769(9,6)$ & $4524(13,8)$ & $2245(6,0)$ & $200(6,1)$ \\
\hline 50 à $<60$ & $11220(16,0)$ & $6264(19,1)$ & $4956(13,2)$ & $435(13,3)$ \\
\hline 60 à < 70 & $14457(20,6)$ & $6075(18,6)$ & $8382(22,3)$ & $773(23,6)$ \\
\hline 70 à < 80 & $13345(19,0)$ & $4324(13,2)$ & $9021(24,0)$ & $946(28,9)$ \\
\hline$\geq 80$ & $14704(20,9)$ & $3984(12,2)$ & $10720(28,6)$ & $682(20,9)$ \\
\hline \multicolumn{5}{|l|}{ Sexe } \\
\hline Féminin & $39213(55,8)$ & $19586(59,8)$ & $19627(52,3)$ & $1584(48,4)$ \\
\hline Masculin & $30042(42,7)$ & $12625(38,6)$ & $17417(46,4)$ & $1643(50,3)$ \\
\hline Inconnu & $1033(1,5)$ & $534(1,6)$ & $499(1,3)$ & $42(1,3)$ \\
\hline \multicolumn{5}{|l|}{ Maladies préexistantes (avant la COVID-19) } \\
\hline Insuffisance rénale aiguë & $3798(5,4)$ & $831(2,5)$ & $2967(7,9)$ & $323(9,9)$ \\
\hline Asthme & $4274(6,1)$ & $2042(6,2)$ & $2232(5,9)$ & $238(7,3)$ \\
\hline Fibrillation ou flutter auriculaires & $5667(8,1)$ & $1528(4,7)$ & $4139(11,0)$ & $381(11,7)$ \\
\hline Dépression ou trouble bipolaire & $11035(15,7)$ & $4139(12,6)$ & $6896(18,4)$ & $481(14,7)$ \\
\hline Infarctus cérébral & $2726(3,9)$ & $664(2,0)$ & $2062(5,5)$ & $138(4,2)$ \\
\hline Maladie rénale chronique & $9856(14,0)$ & $2363(7,2)$ & $7493(20,0)$ & $778(23,8)$ \\
\hline Maladie hépatique chronique & $662(0,9)$ & $195(0,6)$ & $467(1,2)$ & $42(1,3)$ \\
\hline Maladie pulmonaire obstructive chronique & $7424(10,6)$ & $2056(6,3)$ & $5368(14,3)$ & $534(16,3)$ \\
\hline Démence & $8477(12,1)$ & $2335(7,1)$ & $6142(16,4)$ & $323(9,9)$ \\
\hline Diabète de type 1 & $1088(1,5)$ & $361(1,1)$ & $727(1,9)$ & $79(2,4)$ \\
\hline Diabète de type 2 & $19452(27,7)$ & $6446(19,7)$ & $13006(34,6)$ & $1341(41,0)$ \\
\hline Dyslipidémie & $19479(27,7)$ & $7378(22,5)$ & $12101(32,2)$ & $1323(40,5)$ \\
\hline Insuffisance cardiaque & $8072(11,5)$ & $1989(6,1)$ & $6083(16,2)$ & $593(18,1)$ \\
\hline Hypertension essentielle & $28746(40,9)$ & $10333(31,6)$ & $18413(49,0)$ & $1789(54,7)$ \\
\hline Maladie inflammatoire de l'intestin & $413(0,6)$ & $188(0,6)$ & $225(0,6)$ & $28(0,9)$ \\
\hline Cardiopathie ischémique & $8959(12,7)$ & $2445(7,5)$ & $6514(17,4)$ & $679(20,8)$ \\
\hline Cancer & $4184(6,0)$ & $1310(4,0)$ & $2874(7,7)$ & $270(8,3)$ \\
\hline Maladie vasculaire périphérique & $6468(9,2)$ & $1680(5,1)$ & $4788(12,8)$ & $429(13,1)$ \\
\hline Trouble psychotique & $3221(4,6)$ & $926(2,8)$ & $2295(6,1)$ & $135(4,1)$ \\
\hline Maladie rhumatismale & $1918(2,7)$ & $789(2,4)$ & $1129(3,0)$ & $131(4,0)$ \\
\hline Thromboembolie veineuse & $1960(2,8)$ & $529(1,6)$ & $1431(3,8)$ & $129(3,9)$ \\
\hline \multicolumn{5}{|c|}{$\begin{array}{l}\text { Remarque : COVID-19 = maladie à coronavirus } 2019, \text { El = écart interquartile. } \\
\text { *Certaines maladies préexistantes de cette liste figurent sous plus d'une catégorie de codes. Des définitions sont fournies à l'annexe 1, Méthodes. } \\
\text { †Sauf indication contraire. } \\
\text { fLes patients ambulatoires sont ceux qui n'ont pas fait l'objet de demandes de remboursement durant la période à risque à l'égard de la COVID-19. } \\
\text { §Les patients hospitalisés sont ceux qui ont fait l'objet de demandes de remboursement à n'importe quel moment durant la période à risque à l'égard de la COVID-19; ils comprennent } \\
\text { les patients aux soins intensifs. } \\
\text { 9Les patients aux soins intensifs sont ceux qui ont fait l'objet de demandes de remboursement à cet effet, à n'importe quel moment durant la période à risque. }\end{array}$} \\
\hline
\end{tabular}


chroniques après la COVID-19, ce qui confirme que la période de référence plus longue utilisée dans l'analyse principale, soit 90 jours, était nécessaire pour détecter les maladies chroniques déjà présentes avant la COVID-19 (annexe 1, tableau 10). Elle a aussi révélé que la fréquence des codes susceptibles de représenter des problèmes de santé aigus, comme « embolie et thrombose d'autres veines » pouvait augmenter modérément avec la COVID-19 (RC 1,74; IC à $95 \%$ 1,58-1,93), donnant à penser que l'analyse principale a pu passer à côté de certaines maladies aiguës ayant un lien comparativement modeste avec la COVID-19.

\section{Interprétation}

Dans cette étude menée sur plus de 70000 personnes ayant reçu un diagnostic de COVID-19, nous avons constaté que la maladie était associée à un large éventail de complications. Les plus fréquentes que nous avons observées, soit la pneumonie virale, l'insuffisance respiratoire, l'insuffisance rénale aiguë et la sepsie, étaient prévisibles, puisqu'elles ont été bien décrites dans la littérature ${ }^{17-20}$. Nous avons aussi recensé des complications moins courantes qui avaient déjà été décrites dans des séries de cas ou de petites études, comme la coagulation intravasculaire disséminée $e^{21}$, le pneumothorax ${ }^{22}$, la myocardite ${ }^{23}$ et la rhabdomyolyse ${ }^{24}$. Notre étude fournit les estimations du risque absolu et les rapports de cotes pour tous les diagnostics en lien avec la COVID-19, estimations que les professionnels de la santé et les décideurs devraient connaître pour comprendre les risques de complications. Par exemple, la myocardite aiguë présente un RC de 8,17 , mais un risque global de $0,1 \%$, ce qui montre qu'un lien très étroit entre un problème de santé et la COVID-19 ne se traduit pas nécessairement par un risque global élevé.

Étant donné la multiplication des études sur la COVID-19 depuis la description du premier cas de la maladie en 2019, les médecins ont du mal à rester à jour et à faire la distinction entre de fausses affirmations et de réels liens de cause à effet. Les biais de déclaration et de publication peuvent entraîner la surestimation de certaines observations, et les résultats publiés doivent souvent être confirmés par d'autres. L'une des principales forces de notre approche analytique est qu'elle a tenu compte de tous les codes de diagnostic possibles de la CIM-10-MC et simplement quantifié les diagnostics posés après l'infection par le virus de la COVID-19.

Cependant, même si des rapports ont largement fait état d'un lien entre la COVID-19 et un risque plus élevé d'AVC ${ }^{25}$, notre étude n'a pas permis de confirmer ce lien. Pour le code 163 (« infarctus

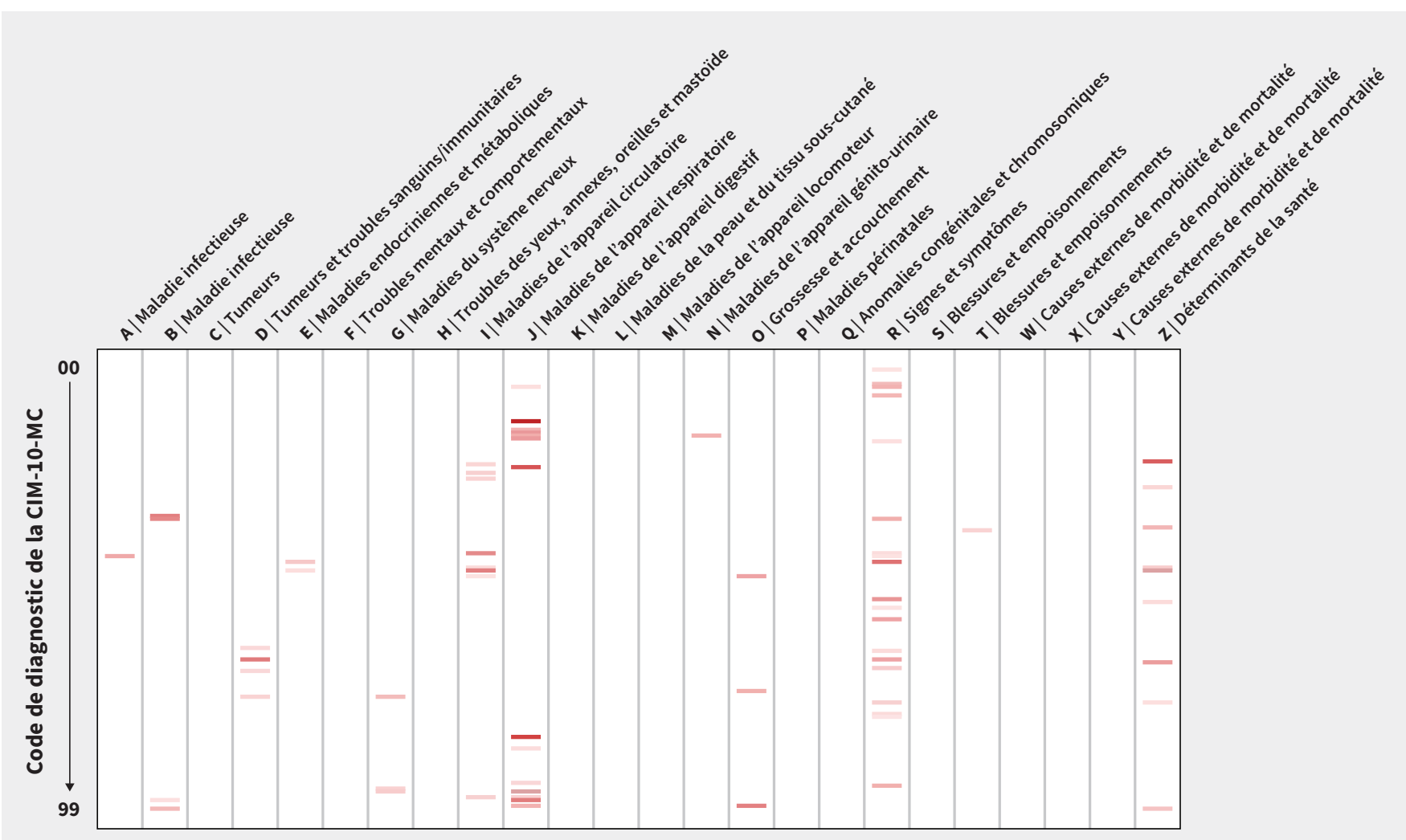

Figure 1 : Carte de densité des complications potentielles de la maladie à coronavirus 2019 (COVID-19), selon les catégories de la Classification internationale des maladies, $10^{e}$ révision, modification clinique (CIM-10-MC). On y trouve les rapports de cotes pour leur lien avec la COVID-19. Les bandes montrent les codes de diagnostic de la CIM-10-MC (regroupés par catégories) significativement associés à la COVID-19 avec un niveau de significativité après correction de Bonferroni et pour lesquels les rapports de cotes étaient supérieurs à 1. Les portions sombres représentent les rapports de cotes plus élevés. Les codes mis en évidence ici sont décrits à la figure 2. 


\begin{tabular}{|c|c|c|c|c|c|}
\hline Type de code & Code de diagnostic de la CIM-10-MC & $\begin{array}{c}\text { Préval. } \\
(\%)\end{array}$ & $\begin{array}{l}\text { Risque } \\
(\%)\end{array}$ & RC (I & $95 \%)$ \\
\hline \multirow{5}{*}{ Maladies infectieuses } & A41 - Autres sepsies & 3,4 & 10,4 & $4,23(4,01-4,46)$ &. \\
\hline & B33 - Autres maladies virales, NCA & 0,0 & 0,2 & $10,88(6,55-18,05)$ & $\because-$ \\
\hline & B34 - Infection virale, siège non précisé & 1,3 & 9,5 & $8,47(7,86-9,12)$ & 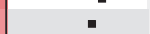 \\
\hline & B96 - Autres agents bactériens, CA & 1,9 & 2,2 & $1,25(1,16-1,35)$ & - \\
\hline & B99 - Maladies infectieuses, autres et non précisées & 0,2 & 0,6 & $2,94(2,42-3,57)$ & - \\
\hline \multirow{4}{*}{$\begin{array}{l}\text { Troubles sanguins/ } \\
\text { immunitaires }\end{array}$} & D61 - Autres anémies aplasiques (aplasies médullaires) & 0,5 & 0,5 & $1,50(1,28-1,75)$ & $=$ \\
\hline & D65 - Coagulation intravasculaire disséminée & 0,0 & 0,1 & $11,83(5,26-26,62)$ & $=$ \\
\hline & D69 - Purpura/autres affections hémorragiques & 1,7 & 2,0 & $1,52(1,40-1,65)$ & - \\
\hline & D72 - Autres anomalies des leucocytes & 2,7 & 4,0 & $1,67(1,57-1,78)$ & \\
\hline \multirow{2}{*}{$\begin{array}{l}\text { Maladies endocriniennes/ } \\
\text { métaboliques }\end{array}$} & E43 - Malnutrition grave, sans précision & 0,8 & 1,1 & $2,11(1,86-2,38)$ &. \\
\hline & E46 - Malnutrition, sans précision & 1,1 & 1,0 & $1,32(1,18-1,48)$ &. \\
\hline \multirow{3}{*}{$\begin{array}{l}\text { Maladies du système } \\
\text { nerveux }\end{array}$} & G72 - Myopathies autres et sans précision & $\frac{1,1}{0,2}$ & 0,4 & $2,70(2,17-3,36)$ & - \\
\hline & G92 - Encéphalopathie toxique & 0,5 & 0,8 & $1,73(1,51-1,98)$ & \\
\hline & G93 - Autres affections du cerveau & 3,6 & 4,9 & $1,93(1,82-2,04)$ & . \\
\hline \multirow{8}{*}{$\begin{array}{l}\text { Maladies de l'appareil } \\
\text { circulatoire }\end{array}$} & 121 - Infarctus aigu du myocarde & 1,5 & 1,8 & $1,59(1,45-1,74)$ & - \\
\hline & 124 - Autres cardiopathies ischémiques aiguës & 0,4 & 0,6 & $1,79(1,53-2,09)$ & $=$ \\
\hline & 126 - Embolie pulmonaire & 0,9 & 1,0 & $1,66(1,47-1,87)$ & 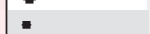 \\
\hline & 140 - Myocardite aiguë & 0,0 & 0,1 & $8,17(3,58-18,62)$ & 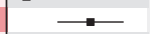 \\
\hline & 145 - Autres troubles de la conduction & 1,1 & 1,3 & $1,39(1,26-1,54)$ & . \\
\hline & 146 - Arrêt cardiaque & 0,1 & 1,1 & $10,43(8,23-13,21)$ & $\Rightarrow$ \\
\hline & 148 - Fibrillation et flutter auriculaires & 8,1 & $\begin{array}{l}1,1 \\
4,6\end{array}$ & $1,19(1,13-1,26)$ & - \\
\hline & 195 - Hypotension & 2,7 & 3,8 & $1,80(1,68-1,92)$ & . \\
\hline \multirow{14}{*}{$\begin{array}{l}\text { Maladies de l'appareil } \\
\text { respiratoire }\end{array}$} & J06 - Infections aiguës des voies respiratoires supérieures & 5,0 & 5,9 & $1,29(1,23-1,36)$ & 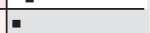 \\
\hline & J12 - Pneumonie virale, NCA & 0,3 & 27,6 & $177,63(147,19-214,37)$ & \\
\hline & J15 - Pneumonie bactérienne, NCA & 0,7 & 2,0 & $3,46(3,09-3,87)$ & . \\
\hline & J16 - Pneumonie causée par d'autres micro-organismes infectieux & 0,1 & 0,4 & $6,26(4,65-8,43)$ & $=$ \\
\hline & J17 - Pneumonie au cours d'autres maladies, CA & 0,0 & 0,1 & $3,83(2,05-7,16)$ & $\rightarrow-$ \\
\hline & J18 - Pneumonie, micro-organisme non précisé & 5,4 & 20,4 & $5,68(5,44-5,94)$ & - \\
\hline & J22 - Infection aiguë des voies respiratoires inférieures, sans précision & 0,2 & 4,4 & $28,77(23,75-34,84)$ & $=$ \\
\hline & J80 - Syndrome de détresse respiratoire aiguë & 0,1 & 4,3 & $41,60(33,00-52,45)$ & \\
\hline & J84 - Autres affections pulmonaires interstitielles & 0,8 & 0,8 & $1,36(1,20-1,55)$ & 1. \\
\hline & J90 - Épanchement pleural, NCA & 2,4 & 2,8 & $1,56(1,45-1,68)$ & . \\
\hline & J93 - Pneumothorax et fuite d'air & 0,1 & 0,4 & $3,38(2,68-4,26)$ & $=$ \\
\hline & J95 - Troubles respiratoires post-intervention & 0,2 & 0,3 & $1,72(1,39-2,12)$ & $=$ \\
\hline & J96 - Insuffisance respiratoire, NCA & 4,6 & 22,6 & $11,36(10,74-12,02)$ & . \\
\hline & J98 - Autres maladies respiratoires & 3,8 & 10,6 & $3,43(3,27-3,60)$ & \\
\hline Maladies de l'appareil génito-urinaire & N17 - Insuffisance rénale aiguë & $\frac{3,0}{5,4}$ & $\frac{11,0}{11,8}$ & 3,50(3,21-34,3,66) & . \\
\hline \multirow{3}{*}{ Grossesse } & O48 - Grossesse prolongée & 0,0 & 0,1 & $4,75(2,25-10,01)$ & \\
\hline & 070 - Déchirure obstétricale du périnée & 0,0 & 0,1 & $3,71(2,08-6,65)$ & 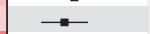 \\
\hline & 098 - Maladies infectieuses de la mère & 0,1 & 0,4 & $9,84(6,86-14,13)$ & $=$ \\
\hline \multirow{19}{*}{ Signes et symptômes } & Ro0 - Anomalies des battements cardiaques & 5,1 & 5,4 & $1,21(1,15-1,27)$ &. \\
\hline & R05 - Toux & 10,4 & 22,0 & $2,79(2,70-2,88)$ & . \\
\hline & R06 - Anomalies de la respiration & 11,9 & 26,2 & $3,42(3,31-3,53)$ & - \\
\hline & R09 - Autres S et S relatifs aux appareils circulatoire et respiratoire & 5,8 & 14,0 & $3,10(2,97-3,23)$ & - \\
\hline & R19 - Autres S et S relatifs à l'appareil digestif et à l'abdomen & 3,9 & 4,4 & $1,26(1,19-1,33)$ & \\
\hline & R34 - Anurie et oligurie & 0,1 & 0,3 & $3,81(2,77-5,24)$ & $=$ \\
\hline & R40 - Somnolence, stupeur et coma & 1,6 & 1,9 & $1,37(1,26-1,48)$ & - \\
\hline & R41 - Autres S et S relatifs aux fonctions cognitives & 8,4 & 7,3 & $1,21(1,16-1,26)$ & . \\
\hline & R43 - Troubles de l'odorat et du goût & 0,0 & 0,6 & $14,62(10,06-21,25)$ & $=$ \\
\hline & R50 - Fièvre d'origine autre et inconnue & 4,8 & 22,2 & $6,52(6,24-6,82)$ & 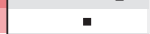 \\
\hline & R53 - Malaise et fatigue & 13,1 & 12,7 & $1,21(1,17-1,25)$ & . \\
\hline & R57 - Choc, NCA & 0,3 & 1,4 & $4,76(4,09-5,54)$ & $=$ \\
\hline & R62 - Retard du développement & 1,2 & 1,1 & $1,44(1,29-1,60)$ & - \\
\hline & R65 - Syndrome de réponse inflammatoire systémique & 1,2 & $\begin{array}{l}1,1 \\
4,9\end{array}$ & $4,83(4,45-5,23)$ & - \\
\hline & R68 - Autres S et S généraux & 3.8 & 6,0 & $2,00(1,89-2,11)$ & \\
\hline & R74 - Anomalie des taux d'enzymes sériques & 1,5 & 2,3 & $1,79(1,65-1,94)$ & - \\
\hline & R78 - Présence de drogues et d'autres substances dans le sang & 1,0 & 1,2 & $1,41(1,27-1,56)$ & - \\
\hline & R79 - Autres résultats anormaux des examens chimiques du sang & 4,0 & 4,2 & $1,15(1,09-1,21)$ & - \\
\hline & R91 - Résultats anormaux d'imagerie diagnostique du poumon & 4,7 & 12,8 & $3,70(3,53-3,87)$ & - \\
\hline Troubles externes & T38 - Intoxications par les hormones, NCA & 0,2 & 0,3 & $1,66(1,35-2,04)$ & $=$ \\
\hline \multirow{9}{*}{$\begin{array}{l}\text { Déterminants } \\
\text { de la santé }\end{array}$} & Z20 - Exposition à des maladies transmissibles & 1,0 & 17,3 & $22,10(20,28-24,08)$ & 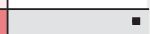 \\
\hline & Z29 - Autres mesures prophylactiques & 0,3 & 0,4 & $1,59(1,31-1,95)$ & * \\
\hline & Z37 - Issue de la grossesse & 0,1 & 0,3 & $3,19(2,45-4,16)$ & 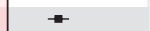 \\
\hline & Z45 - Ajustement et entretien d'un dispositif implantable & 1,7 & 2,8 & $1,89(1,75-2,04)$ & - \\
\hline & Z46 - Mise en place et ajustement d'autres appareils & 1,3 & 3,7 & $3,38(3,12-3,66)$ & - \\
\hline & Z51 - Autre consultation pour soins médicaux & 3,2 & 3,7 & $1,43(1,35-1,52)$ & \\
\hline & Z66 - Ordre de non-réanimation & 0,7 & 2,8 & $5,51(4,92-6,17)$ & - \\
\hline & Z74 - Difficulté liée à une dépendance envers la personne qui donne les soins & 7,8 & 6,7 & $1,28(1,22-1,34)$ & - \\
\hline & Z99 - Dépendance envers des appareils, NCA & 6,0 & 7,3 & $2,38(2,26-2,50)$ & - \\
\hline
\end{tabular}

Figure 2 : Codes de diagnostic identifiés comme complications potentielles de la maladie à coronavirus 2019 (COVID-19) chez tous les patients. Les prévalences de base (Préval.), les risques globaux (Risque) et les rapports de cotes (RC) avec des intervalles de confiance (IC) à $95 \%$ pour le lien avec la COVID-19 sont présentés pour les codes de diagnostic de la Classification internationale des maladies, 10 révision, modification clinique (CIM-10-MC), regroupés par catégories. La prévalence de base est la proportion de patients présentant le problème de santé durant la période de référence parmi tous les patients. Le risque global est la proportion de patients présentant le problème de santé après avoir contracté la COVID-19 parmi les patients qui ne souffraient pas déjà de ce problème de santé. Seuls les codes significativement associés $(R C>1)$ sont présentés. Le seuil de significativité pour cette analyse était de 0,05/(nombre total de codes analysés) $=0,05 / 1724=2,90^{\mathrm{E}-05}$. Les données complètes sont fournies à l'annexe 1 , tableau 2 (accessible en anglais au www.cmaj.ca/lookup/doi/10.1503/cmaj.201686/tab-related-content). Les calculs sont expliqués à l'annexe 1, figure 3. L'axe des abscisses montre les RC représentés sur une échelle logarithmique. Les portions sombres représentent les valeurs plus élevées. Remarque : CA = classé ailleurs, NCA = non classé ailleurs, $\mathrm{S}$ et $\mathrm{S}=$ signes et symptômes. 
A

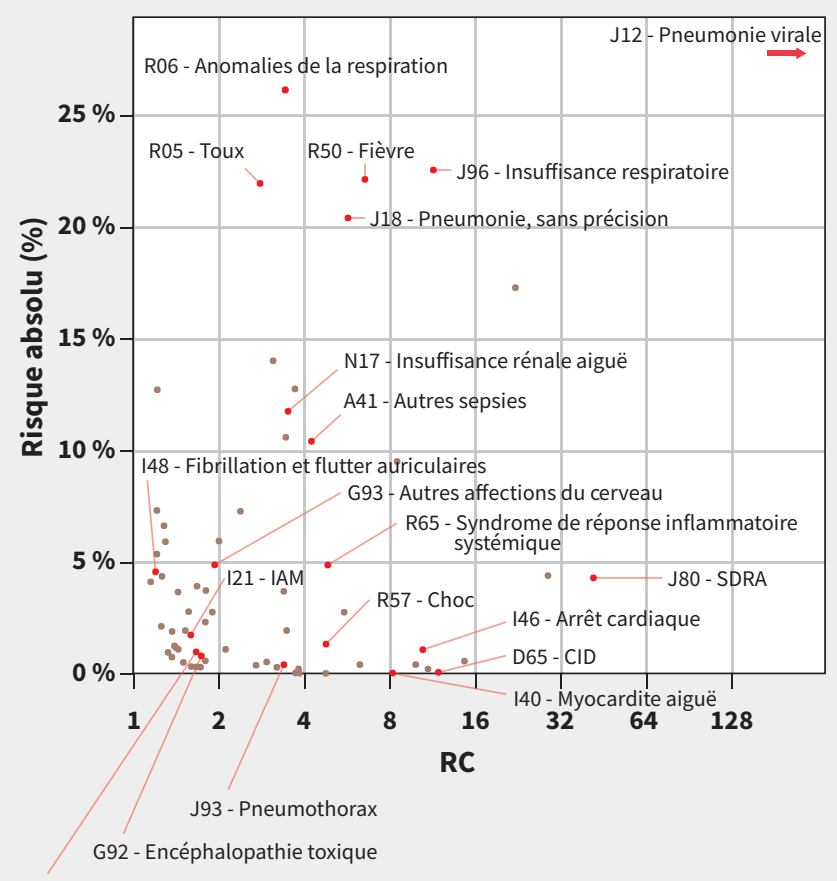

126 - Embolie pulmonaire
Patients aux soins intensifs

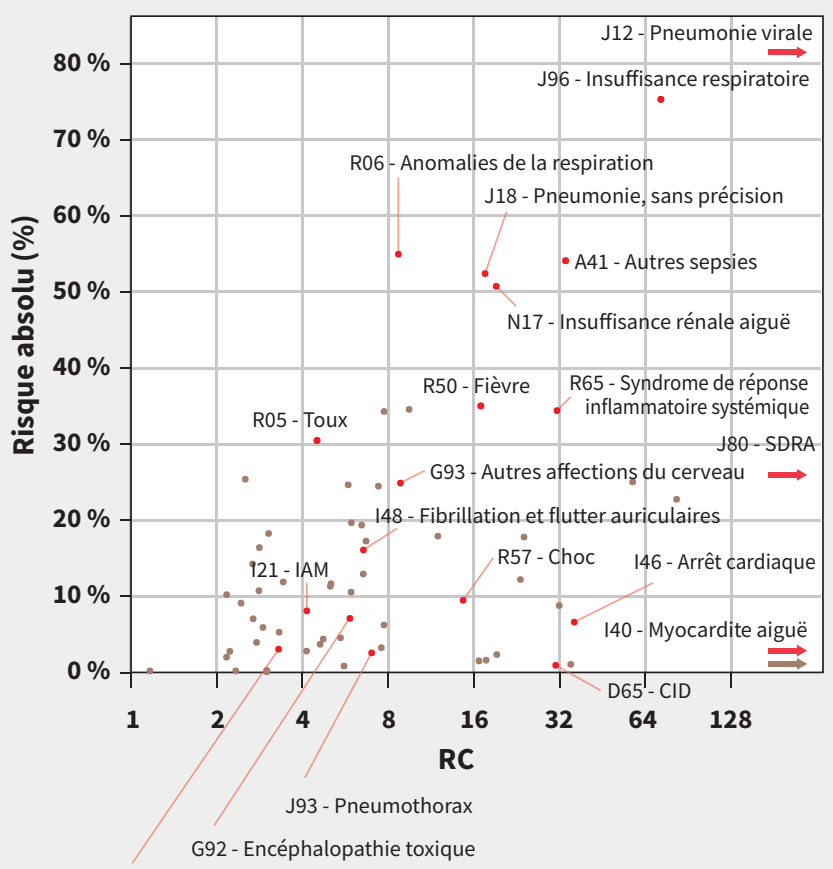

126 - Embolie pulmonaire

Figure 3 : Risques et rapports de cotes (RC) des complications de la maladie à coronavirus 2019 (COVID-19) repérées. Un graphique des complications potentielles de la COVID-19 repérées dans l'analyse principale est présenté ici. L'axe des abscisses montre les RC sur une échelle logarithmique, représentant l'étroitesse du lien entre la COVID-19 et la complication citée. L'axe des ordonnées montre le risque global pour la complication (c.-à-d., la fréquence du problème de santé après l'acquisition de la COVID-19 chez les patients qui n'en étaient pas atteints auparavant). Les rapports de cotes et les estimations du risque ont été calculés dans les populations respectives (soit [A] tous les patients ou [B] les patients aux soins intensifs). Les flèches montrent les codes de diagnostic de la Classification internationale des maladies, 10évision, modification clinique (CIM-10-MC) dont le RC excède la limite de l'échelle logarithmique. Par manque d'espace, seules certaines complications représentant différents éventails de RC et de risques sont mises en évidence; les données complètes sont fournies à la figure 2 . Remarque : $C I D=$ coagulation intravasculaire disséminée, IAM = infarctus aigu du myocarde, NCA = non classé ailleurs, SDRA = syndrome de détresse respiratoire aiguë, $\mathrm{S}$ et $\mathrm{S}$ = signes et symptômes.

cérébral ») dans notre population générale, nous avons obtenu un $\mathrm{RC}$ de 0,58 et un risque global de $1,5 \%$ (annexe 1 , tableau 3), ce qui donne à penser que, si les patients atteints de COVID-19 présentent une fréquence significative d'AVC, le lien causal avec la COVID-19 ne se confirme pas dans cette population. Le syndrome inflammatoire multisystémique chez les enfants n'a pas pu être étudié directement car aucun code spécifique ne lui a été attribué dans la CIM-10-MC, même si aucun lien n'a été noté pour la maladie similaire de Kawasaki, portant le code M30.3.

Nous avons aussi observé que le risque de nombreux codes de diagnostic a semblé diminuer après l'infection par le virus de la COVID-19. Nous avançons 2 explications possibles. Durant les stades initiaux du traitement de la COVID-19, de nombreuses maladies chroniques et d'autres problèmes de santé moins graves peuvent avoir été jugés moins prioritaires et n'ont donc pas figuré dans les réclamations. De plus, pour les maladies aiguës, la période de référence ( 90 jours) pourrait cumuler plus d'événements que la période de risque (38 jours) en raison de sa durée plus longue. Cela pourrait expliquer pourquoi, par exemple, le risque d'infarctus cérébral a semblé diminuer après le déclenchement de la COVID-19 dans notre analyse principale, tout en augmentant légèrement dans notre analyse de sensibilité, avec une période de référence plus brève.

Au moment d'interpréter nos estimations du risque, il est important de garder en tête que des maladies plus bénignes ou aux manifestations cliniques plus discrètes ne figurent pas d'emblée dans les données de réclamations. Cela pourrait expliquer le fait que, même si nous avons observé des liens étroits entre la COVID-19 et certaines complications telles que la toux et les troubles de l'odorat ou du goût, les estimations du risque global de ces complications $(22,0 \%$ et $0,6 \%$, respectivement, dans la population générale) ont été substantiellement moindres que dans les rapports publiés ( $79 \%$ et $65 \%$, respectivement) ${ }^{9,26}$. Ainsi, les observations relatives aux codes de maladies relativement bénignes reflèteraient peut-être mieux l'étroitesse du lien que les estimations du risque absolu. En revanche, les estimations du risque de troubles graves et manifestes reflèteront plus probablement le risque réel puisque ces maladies auront plus de chance d'être traitées ou portées à l'attention des professionnels de la santé et, par conséquent, de 


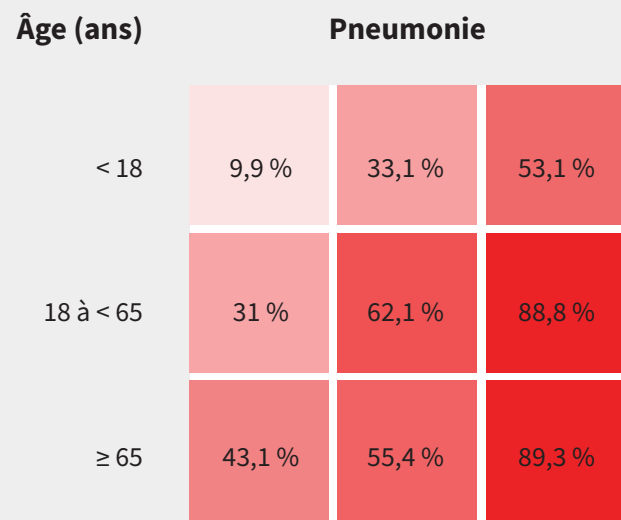

Insuffisance respiratoire ou SDRA

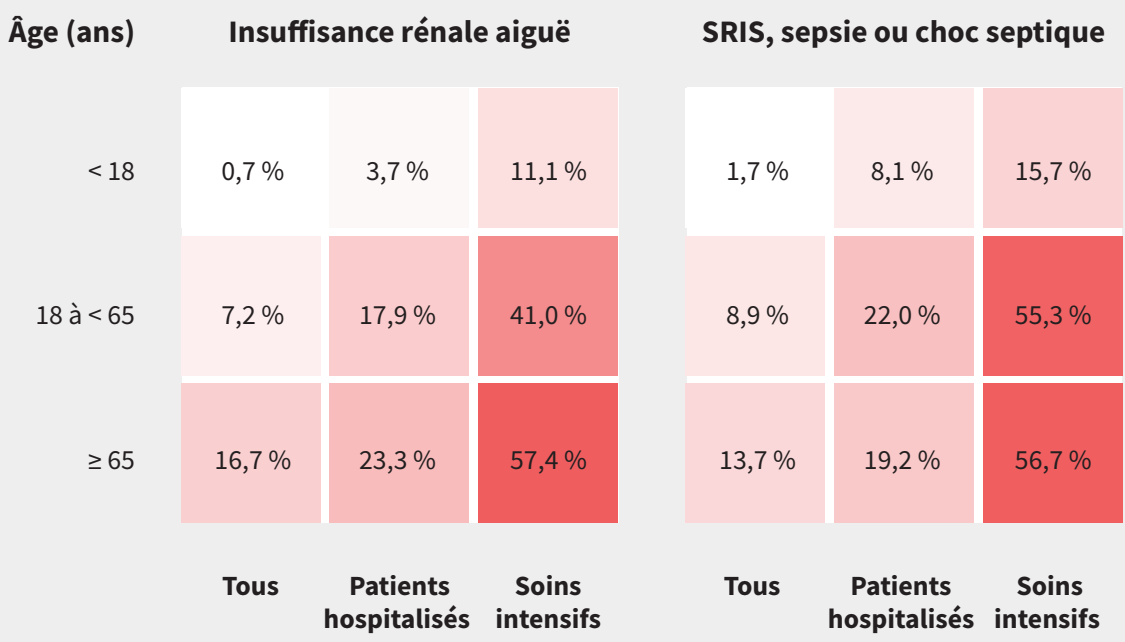

Figure 4 : Estimations du risque des plus fréquentes complications de la maladie à coronavirus 2019 (COVID-19), selon l'âge et le statut d'hospitalisation. Certains de ces problèmes de santé se retrouvent dans plus d'une catégorie de codes de la Classification internationale des maladies, $10^{e}$ révision, modification clinique (CIM-10-MC). Pneumonie : désignée par le code J12 (pneumonie virale non classée ailleurs) ou J18 (pneumonie, micro-organisme non précisé). Insuffisance respiratoire ou syndrome de détresse respiratoire aiguë (SDRA) : désignés par le code J96 (insuffisance respiratoire non classée ailleurs) ou J80 (syndrome de détresse respiratoire aiguë). Insuffisance rénale aiguë : désignée par le code N17 (insuffisance rénale aiguë). Syndrome de réponse inflammatoire systémique (SRIS), sepsie ou choc septique : désignés par le code A41 (autres sepsies) ou R65 (syndrome de réponse inflammatoire systémique). Les données complètes pour ces observations se trouvent à l'annexe 1, tableau 6 (accessible en anglais au www.cmaj.ca/lookup/doi/10.1503/cmaj.201686/tab-related-content).

figurer dans les données de réclamations ${ }^{27}$. Par exemple, nos estimations des risques d'insuffisance respiratoire $(40,0 \%)$ et d'insuffisance rénale aiguë $(21,2 \%)$ chez les patients hospitalisés sont similaires aux estimations antérieures (54\% et $15 \%$, respectivement $)^{20}$. Une autre mise en garde concernant l'interprétation de nos estimations du risque est qu'elles calculent uniquement le risque de maladie nouvellement diagnostiquée et non le risque d'exacerbation par la COVID-19 d'une maladie préexistante. En outre, nos estimations ne reflètent que le risque chez les patients qui consultent pour la COVID-19.

Cette étude a comme principaux atouts son volumineux échantillon et son approche centrée sur les données. Elle tire aussi sa force de son modèle d'auto-appariement pré- et post- exposition qui permet de tenir compte des variables de confusion en appariant les observations pré- et post-diagnostic de COVID-19 ${ }^{12}$. L'utilisation des dossiers de réclamations des patients hospitalisés et ambulatoires nous a permis de dresser des antécédents médicaux relativement complets pour chaque patient et de tenir compte des problèmes de santé déjà présents avant le diagnostic de COVID-19.

\section{Limites de l'étude}

Une des limites des données de réclamations est que nous avons repéré les cas de COVID-19 au moyen des codes de diagnostic. Même si les codes que nous avons utilisés font état de cas confirmés de COVID-19 $9^{10,11}$, il est possible que certains patients aient été 
mal catégorisés. De plus, le caractère récent de la pandémie de COVID-19 nous a forcés à utiliser des données de réclamations non réglées qui pourraient n'avoir pas été traitées au moment de la saisie et avoir été ultérieurement contestées par un payeur. Certaines données pourraient par conséquent être mal classées, ce qui nuit à la précision des estimations. Par ailleurs, les codes de la CIM10-MC ne sont pas nécessairement des définitions validées des maladies et ont été assignés par des professionnels de la santé qui travaillent dans différents milieux de soins un peu partout aux États-Unis; on ne peut donc pas se fier à la constance et à la fiabilité des notions que recouvrent ces codes pour les maladies visées. Cependant, étant donné la taille de l'ensemble de données, nous croyons que ces enjeux n'ont pas exercé d'influence majeure sur nos résultats. En outre, étant donné que les données de la période de référence (données antérieures) risquent d'être plus complètes que celles de la période de risque, les RC peuvent avoir été sousestimés, entraînant de ce fait un biais généralement conservateur dans la détection des complications de la COVID-19. Comme les résultats de cette étude concordent largement avec les complications connues de la COVID-19, nous croyons que cela n'a pas significativement affecté nos résultats.

Les milieux de soins représentés dans la base de données ne sont pas exclusivement des établissements hospitaliers universitaires où sont traités les cas de COVID-19 les plus graves; nos résultats pourraient donc exclure certains de ces cas les plus graves. Une autre limite quant à la capacité d'extrapoler les résultats est que les patients devaient avoir présenté au moins 1 réclamation pour frais médicaux et qu'ils étaient peut-être plus malades que la population générale durant la période de référence. Par conséquent, les estimations des risques présentées ici sont peut-être supérieures aux risques dans la population générale, car les patients étudiés étaient plus susceptibles d'avoir des comorbidités. En terminant, certaines des complications relevées pourraient ne pas être des conséquences directes de l'infection au SRAS-CoV-2, mais plutôt des manifestations iatrogènes (dues aux traitements).

\section{Conclusion}

Dans l'ensemble, les complications le plus fréquemment associées à la COVID-19 chez les patients qui consultent sont la pneumonie, l'insuffisance respiratoire, l'insuffisance rénale et la sepsie ou la réponse inflammatoire systémique. Après avoir analysé tous les codes de diagnostic possibles, nous confirmons que la COVID19 est aussi associée à toute une gamme d'autres problèmes (cardiaques, thrombotiques, etc.), même si les risques globaux de la plupart d'entre eux sont comparativement faibles. Comprendre la gamme complète des affections associées à la COVID-19 peut faciliter le pronostic, orienter les décisions thérapeutiques et améliorer l'information donnée aux patients quant au risque réel des complications rapportées dans la littérature et les médias.

\section{Références}

1. WHO coronavirus disease (COVID-19) dashboard. Geneva: World Health Organization; 2020. Accessible ici : covid19.who.int/ (consulté le 20 nov. 2020).

2. Huang $C$, Wang $Y$, Xingwang $L$, et al. Clinical features of patients infected with 2019 novel coronavirus in Wuhan, China. Lancet 2020;395:497-506.
3. Interim clinical guidance for management of patients with confirmed coronavirus disease (COVID-19). Atlanta: Centers for Disease Control and Prevention; 2020. Accessible ici : www.cdc.gov/coronavirus/2019-ncov/hcp/clinical-guidance -management-patients.html (consulté le 1 juil. 2020).

4. Varga Z, Flammer AJ, Steiger $P$, et al. Endothelial cell infection and endotheliitis in COVID-19. Lancet 2020;395:1417-8.

5. Klok FA, Kruip MJHA, van der Meer NJM, et al. Incidence of thrombotic complications in critically ill ICU patients with COVID-19. Thromb Res 2020;191:145-7.

6. Long B, Brady WJ, Koyfman A, et al. Cardiovascular complications in COVID-19. Am J Emerg Med 2020;38:1504-07.

7. Chiotos K, Bassiri H, Behrens EM, et al. Multisystem inflammatory syndrome in children during the COVID-19 pandemic: a case series. J Pediatric Infect Dis Soc 2020;9:393-8.

8. Mao L, Jin H, Wang M, et al. Neurologic manifestations of hospitalized patients with coronavirus disease 2019 in Wuhan, China. JAMA Neurol 2020;77:683-90.

9. Spinato G, Fabbris C, Polesel J. Alterations in smell or taste in mildly symptomatic outpatients with SARS-CoV-2 infection. JAMA 2020;323: 2089-2090.

10. ICD-10-CM official coding guidelines - supplement: coding encounters related to COVID-19 coronavirus outbreak: effective February 20, 2020. Atlanta: Centers for Disease Control and Prevention; 2020. Accessible ici : www.cdc.gov/ nchs/data/icd/ICD-10-CM-Official-Coding-Gudance-Interim-Advice-coronavirus

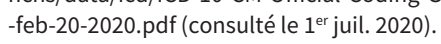

11. ICD-10-CM official coding and reporting guidelines April 1, 2020 through September 30, 2020. Atlanta: Centers for Disease Control and Prevention; 2020. Accessible ici : www.cdc.gov/nchs/data/icd/COVID-19-guidelines-final.pdf

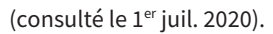

12. Redelmeier DA. The exposure-crossover design is a new methods for studying sustained changes in recurrent events. J Clin Epidemiol 2013;66:955-63.

13. Fay MP. Exact McNemar's Test and matching confidence intervals. 2016. Accessible ici : cran.r-project.org/web/packages/exact2×2/vignettes/exactMcNemar.

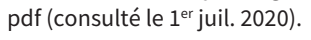

14. Hirsch JA, Nicola G, McGinty G, et al. ICD-10: history and context. AJNR Am J Neuroradiol 2016;37:596-9.

15. ICD-10-CM official guidelines for coding and reporting. Baltimore: Centers for Medicare and Medicaid Services; 2020. Accessible ici : www.cms.gov/Medicare/ Coding/ICD10/Downloads/2020-Coding-Guidelines.pdf (consulté le $1^{\text {er }}$ juil. 2020).

16. Bland JM, Altman DG. Multiple significance tests: the Bonferroni methods. BMJ 1995;310:170.

17. Li X, Ma X. Acute respiratory failure in COVID-19: Is it "typical" ARDS? Crit Care 2020;24:198.

18. Richardson S, Hirsch JS, Narasimhan M. Presenting characteristics, comorbidities, and outcomes among 5700 patients hospitalized with COVID-19 in the New York City area. JAMA 2020;323:2052-9.

19. Goyal P, Choi JJ, Pinheiro LC, et al. Clinical characteristics of COVID-19 in New York City. N Engl J Med 2020;382:2372-4.

20. Zhou F, Yu T, Du R, et al. Clinical course and risk factors for mortality of adult inpatients with COVID-19 in Wuhan, China: a retrospective cohort study. Lancet 2020;395:1054-62.

21. Tang N, Li D, Wang X, et al. Abnormal coagulation parameters are associated with poor prognosis in patients with novel coronavirus pneumonia. $J$ Thromb Haemost 2020;18:844-7.

22. Rohailla S, Ahmed N, Gough K. SARS-CoV-2 infection associated with spontaneous pneumothorax. CMAJ 2020;192:E510.

23. Ruan Q, Yang K, Wang W, et al. Clinical predictors of mortality due to COVID-19 based on an analysis of data of 150 patients from Wuhan, China. Intensive Care Med 2020;46:846-8.

24. Jin M, Tong $\mathrm{Q}$. Rhabdomyolysis as potential late complication associated with COVID-19. Emerg Infect Dis 2020;26:1618-20.

25. Fridman S, Bullrich MB, Jimenez-Ruiz A, et al. Stroke risk, phenotypes, and death in COVID-19: Systematic review and newly reported cases. Neurology 2020;Sep 15 [Cyberpublication avant impression]. doi: 10.1212/WNL.0000000000010851.

26. Chen HJ, Qiu J, Wu B, et al. Early chest CT features of patients with 2019 novel coronavirus (COVID-19) pneumonia: relationship to diagnosis and prognosis. Eur Radiol 2020;30:6178-85.

27. Guidance for industry and FDA staff: best practices for conducting and reporting pharmacoepidemiologic safety studies using electronic healthcare data. Silver Spring (MD): US Food and Drug Administration, Center for Drug Evaluation and Research; 2013. 
Intérêts concurrents : William Murk est consultant pour Aetion, Inc., où il détient également des actions. Monica Gierada, Andrew Weckstein et Jeremy Rassen sont des employés d'Aetion, Inc., où ils détiennent aussi des options d'achat d'actions ou des actions. Reyna Klesh est une employée de HealthVerity, Inc., qui a fourni les données pour cette étude. Aucun autre intérêt concurrent déclaré.

Cet article a été révisé par des pairs.

Affiliations : Faculté de médecine et de sciences biologiques (Murk), Université de Buffalo, Buffalo, N.Y.; Aetion Inc. (Murk, Gierada, Weckstein, Rassen), New York, N.Y.; Département de médecine (Fralick), Université de Toronto, Toronto, Ont.; HealthVerity Inc. (Klesh), Philadelphie, Penn.

Collaborateurs : William Murk a conçu l'étude. Tous les auteurs ont collaboré à la modélisation de l'étude. William Murk, Monica Gierada et Andrew Weckstein ont contribué à l'acquisition, à l'analyse et à l'interprétation des données. Tous les auteurs ont participé à la rédaction du manuscrit, en ont révisé de façon critique le contenu intellectuel important, ont donné leur approbation finale pour la version destinée à être publiée, et assument l'entière responsabilité de tous les aspects du travail. William Murk, Monica Gierada et Michael Fralick ont contribué à parts égales à l'article.

Financement : Cette étude a été financée par Aetion, Inc., un fabricant de logiciels d'analyse de données réelles. Michael Fralick est titulaire d'une bourse Banting et Best des Instituts de recherche en santé du Canada.
Partage des données : Des ententes sur le partage des données empêchent la publication de données à l'échelle des patients. Les données agrégées sur les nombres de patients en fonction des codes diagnostiques qui ne figurent pas déjà dans le supplément sont disponibles sur demande auprès des auteurs.

Propriété intellectuelle du contenu : Il s'agit d'un article en libre accès distribué conformément aux modalités de la licence Creative Commons Attribution (CC BY-NC-ND 4.0), qui permet l'utilisation, la diffusion et la reproduction dans tout médium à la condition que la publication originale soit adéquatement citée, que l'utilisation se fasse à des fins non commerciales (c.-à-d., recherche ou éducation) et qu'aucune modification ni adaptation n'y soit apportée. Voir : https://creativecommons. org/licenses/by-nc-nd/4.0/deed.fr.

Remerciements : Les auteurs remercient Emily Rubinstein, Lacey Gavala, Lakshmi Gonji et Kate Allred pour leur aide concernant les données utilisées dans cette étude. Ils remercient aussi Sebastian Schneeweiss pour ses suggestions utiles au sujet du manuscrit.

Accepté : Le 26 novembre 2020

Correspondance : William Murk, wmurk@buffalo.edu 